\title{
Sarcoidosis and Pain Caused by Small-Fiber Neuropathy
}

\author{
Lara Heij, ${ }^{1}$ Albert Dahan, ${ }^{1}$ and Elske Hoitsma ${ }^{2}$ \\ ${ }^{1}$ Department of Anesthesiology, Leiden University Medical Center, P5Q, Postbus 9600, 2300 RC Leiden, The Netherlands \\ ${ }^{2}$ Department of Neurology, Diaconessenhuis Leiden, Leiden, The Netherlands
}

Correspondence should be addressed to Lara Heij, 1.r.heij@lumc.nl

Received 29 June 2012; Accepted 1 October 2012

Academic Editor: Jeffrey J. Borckardt

Copyright ( 92012 Lara Heij et al. This is an open access article distributed under the Creative Commons Attribution License, which permits unrestricted use, distribution, and reproduction in any medium, provided the original work is properly cited.

\begin{abstract}
Sarcoidosis is a chronic inflammatory illness and small-fiber neuropathy (SFN) is one of the disabling and often chronic manifestations of the disease. SFN presents with peripheral pain and symptoms of autonomic dysfunction. The character of the pain can be burning or shooting. Besides, allodynia and hyperesthesia can exist. Diagnosis is usually made on the basis of clinical features, in combination with abnormal specialized tests. The aim of treatment is often to reduce pain; however, total pain relieve is seldom achieved. The role of TNF- $\alpha$ in the pathogenesis of SFN in sarcoidosis appears interesting to explore. Novel therapeutic agents such as ARA 290, a nonhematopoietic erythropoietin analogue with potent anti-inflammatory and tissue protective properties, are interesting to explore in the treatment of SFN in sarcoidosis.
\end{abstract}

\section{Sarcoidosis}

Sarcoidosis has been known for more than 100 years and has been first described by the dermatologist Hutchinson and several years later by two other dermatologists, Besnier and Boeck. It is a multiorgan inflammatory disorder that is characterized by noncaseating granuloma (Figure 1). The exact etiology remains unknown. It is suspected that exposure to one or more extrinsic antigens in a genetically susceptible individual leads to the overactivation of inflammatory pathways that promote the formation of sarcoid granuloma [1]. Granuloma formation is regulated by a complex interaction between T-helper lymphocytes and macrophages, in which cytokines such as tumor necrosis factor (TNF)- $\alpha$ play an important role.

The clinical course of sarcoidosis is highly variable and depends on ethnicity, duration of illness, site and extension of organ involvement, and activity of the granulomatous process, which shows a tendency to wax and wane. Mode of presentation varies from asymptomatic, to an "acute onset" presenting as Lofgren's syndrome and to a chronic course, frequently accompanied with pain and fatigue. Practically every organ can be involved. However, most commonly $(>90 \%)$ the lungs are affected $[2,3]$. Often patients suffer from symptoms a long time before the diagnosis sarcoidosis is confirmed. Due to the manifold presentation of the disease, it is a challenge to recognize in an early phase. The acute stage of disease usually presents itself with erythema nodosum, arthritis, fever, and fatigue with a good prognosis. Spontaneous remission usually occurs within two years, while chronic sarcoidosis mostly has an insidious onset with often relapses, resolution being less likely. In some of the cases, the disease is progressive. Development of lung fibrosis, cardiac sarcoidosis, and neurosarcoidosis is related to worse prognosis. Factors that trigger the formation of fibrosis in sarcoidosis are poorly understood. Up to $5 \%$ will eventually die from sarcoidosis.

In chronic sarcoidosis, pain and fatigue are important symptoms, even when sarcoidosis is clinically in remission fatigue and pain may persist and become a chronic complaint. These complaints often result in a severe reduction in quality of life. Although a lot of research has been done, the exact mechanism behind this "postsarcoidosis chronic fatigue syndrome" remains unsolved.

Recently, it has been shown that pain in patients with sarcoidosis is often related to neuropathy of small fibers of the peripheral nervous system [4-7].

\section{Small Fiber Neuropathy}

Small-fiber neuropathy (SFN) is a peripheral nerve disorder that selectively affects thinly myelinated $\mathrm{A} \delta$ fibers 


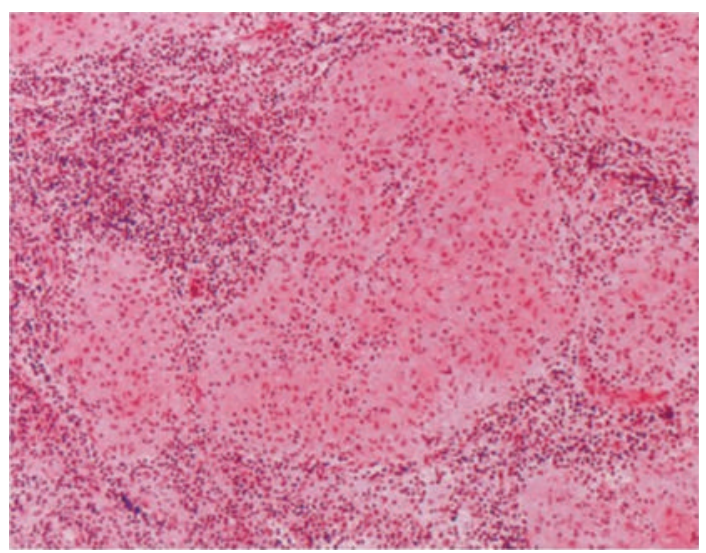

FIgURE 1: A microscopical section of mediastinal lymph node with HE stain, $\times 40$. Multiple granulomas with various sizes from 0,2 to $0,8 \mathrm{~mm}$ in diameter are observed in the lymph node. These granulomas consist of histiocytes, which have large cytoplasm and partly connect to each other but lack a necrotic region.

and unmyelinated C fibers. Small nerve fibers are involved in both somatic and autonomic function [8]. As a result, patients with SFN may present with symptoms of neuropathic pain (NP) and autonomic dysfunction [5].

Damage to or loss of small somatic nerve fibers results in burning pain, tingling, or numbness that typically affects the limbs in a distal to proximal gradient. Symptoms are usually worse at night and often affect sleep. People sometimes sleep with the feet uncovered because they can not bear the touch of the sheets. Besides, walking may be difficult due to pain by the pressure on the floor. When autonomic fibers are affected, patients may experience dry eyes, dry mouth, orthostatic dizziness, constipation, bladder incontinence, sexual dysfunction, hyperhidrosis or hypohidrosis, or red or white skin discoloration. Finally restless legs syndrome may be present, characterized by disagreeable leg sensations that usually occur prior to sleep onset and cause an almost irresistible urge to move (Table 1).

Most patients suffer from length-dependent small-fiber neuropathy (LD-SFSN): symptoms and signs start to develop in the toes and feet, symptoms gradually progress to involve distal legs, fingertips, and hands. Non-length-dependent small-fiber neuropathy (NLD-SFSN) is not as common as LD-SFSN and patients develop complaints in a patchydistribution. This can include face, upper limbs, or trunk before the lower limbs are affected. The NLD-SFSN is more seen in women and presents at a younger age $[9,10]$.

2.1. Diagnosis of Small Fiber Neuropathy. Nerve conduction studies, which are the key in evaluation of other (large fiber) neuropathies, are generally normal in SFN. Therefore, the syndrome of SFN has been an enigma to practitioners because of the unexplained contrast between severe pain and a paucity of neurological and electrophysiological findings. Recent advantages in diagnostic techniques facilitate objective confirmation of clinical diagnosis and characterization of fiber involvement. However, a golden standard for the diagnosis of SFN is not available yet. Diagnosis is usually
TABLE 1: Symptoms of small fiber neuropathy.

\begin{tabular}{ll}
\hline Sensory symptoms & Pain* \\
& Paraesthesias \\
& Sheet intolerance \\
& Restless legs syndrome** \\
Symptoms of autonomic dysfunction & Hypo- or hyperhidrosis \\
& Diarrhoea or constipation \\
& Urinary incontinence or \\
& -retention \\
& Gastroparesis \\
& Sicca syndrome \\
& Blurry vision \\
& Facial flushes \\
& Orthostatic intolerance \\
Sexual dysfunction
\end{tabular}

* Pain in small fiber neuropathy is often burning, tingling, shooting, or prickling in character.

** Restless legs syndrome is a disorder characterized by disagreeable leg sensations that usually occur prior to sleep onset and that cause an almost irresistible urge to move.

made on the basis of clinical features, in combination with abnormal specialized tests, which include among others, assessment of intraepidermal nerve fiber density (IENFD) in skin biopsy, temperature sensation tests, and sudomotor and cardiovagal testing for autonomic fibers $[4,6,11]$. However, all tests have their limitations.

Quantitive sensory testing (QST) includes temperature threshold testing. Thermal (cold and warm) and mechanical (tactile and vibration) detection thresholds assess small-fiber function (including the central pathways). The cold detection threshold (CDT) examines the A-delta-fiber function, while assessment of C-fibre function is examined by the warm detection threshold (WDT). The major limitation of QST is its psychophysical character. As a consequence, malingering and other nonorganic factors can influence test results [12].

To objectively test small nerve fibers, laser-evoked potentials (LEP) and contact heat-evoked potentials (CHEPs), have been developed. It is well established that both laser and contact heat stimulation activate thermo-nociceptive cutaneous nerves. Even though attention and other cognitive processes influence the amplitude of Laser Evoked Potentials (LEPs) and Contact Heat Evoked Potentials (CHEPs) these tests carry up relevant information on the functional state of nociceptive terminals.

For the CHEPs, a thermofoil thermode stimulator is used to reach a temperature of $53^{\circ} \mathrm{C}$ at a rate of $70^{\circ} \mathrm{C} / \mathrm{s}$. It has been shown that patients with sensory neuropathy have loweramplitude CHEPs, which correlates with other SFN tests [13].

Multiple studies have emphasized the importance of intraepidermal nerve fiber density (IENFD) assessment using PGP-9.5 immunofluorescent staining in skin biopsy in the evaluation SFN [14]. Epidermal nerves are the distal terminals of small dorsal root ganglia neurons that pierce 


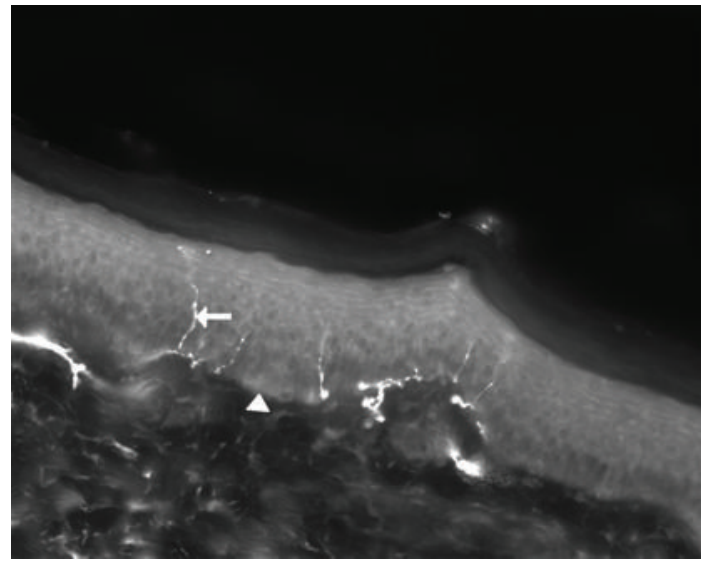

Figure 2: Magnification 200x. Punch skin biopsy from a healthy control showing intraepidermal nerve fibers. Arrow: intraepidermal nerve fiber. Arrowhead: basal membrane (above the basal membrane the epidermis is shown, under the basal membrane the dermis is shown).

the dermal-epidermal basement membrane and penetrate the epidermis. The discovery of the antibody to the neuropeptide protein gene product (PGP) 9.5 made it possible to effectively stain nerve fibers (Figure 2). PGP 9.5 is a ubiquitin C-terminal hydrolase and is enriched in epidermal nerve fibers [14]. A punch biopsy is performed following established procedures, mostly $10 \mathrm{~cm}$ above the lateral malleolus after local anesthesia with $1 \%$ lidocaine. A limitation of skin biopsies is that they are available in only a few academic centers. The histological technique is moderately complicated and time consuming, and before implementing it, a relatively large subset of healthy controls should be studied as the normative range is wide. Finally skin biopsy appears to have a high specificity but low sensitivity in sarcoidosis: Bakkers in 2009 showed that $32,8 \%$ of sarcoidosis patients with symptoms of SFN had a reduced IENFD score in the skin biopsy, and $14,3 \%$ in patients without SFN symptoms had a reduced IENFD [4]. The rule "physicians, not tests make diagnosis" appears especially applicable for SFN. Examination often reveals allodynia, hyperalgesia, or reduced pinprick and thermal sensation in the affected area. Motor strength en proprioception, however, are (as functions of the large fibers) preserved.

2.2. Etiology of Small Fiber Neuropathy. In $50 \%$ of the cases presenting with SFN no underlying disease is found: "idiopathic SFN" [15]. Recent studies have shown gain of function mutations in sodium channel $\mathrm{Na}(\mathrm{V}) 1.7$ in a subset $(28.6 \%)$ of those patients with idiopathic SFN [16]. The exact role of these mutations is unresolved yet.

In $50 \%$ of the cases presenting with SFN, an underlying disease is present, including diabetes, sarcoidosis, and amyloidosis among others (Table 2) [6]. It is remarkable that SFN appears frequent in several immune-mediated diseases. This leads to the hypothesis that there might be a common pathway in immune-mediated diseases resulting in SFN. The idea of an immune-mediated mechanism as the cause of SFN has also been reported by others [17-19].
TABLE 2: Causes of small fiber neuropathy [6].

\begin{tabular}{ll}
\hline Idiopathic & \\
\hline & Familial amyloidosis \\
& Autosomal recessive hereditary neuropathy \\
& Hereditary sensory and autonomic neuropathy \\
Inherited & Fabry's disease \\
& Ross syndrome \\
& Friedreich's ataxia \\
& Tangier disease \\
& Diabetes mellitus \\
& Impaired glucose tolerance \\
& Alcoholism \\
& Systemic amyloidosis \\
& Vasculitis \\
& Sarcoidosis \\
& Sjögren's disease \\
Systemic lupus erythematosus & Guillain-Barre syndrome \\
& Antecedent viral infection \\
& HIV \\
& Antisulfatide antibodies \\
& Hyperlipidemia \\
& Complex regional pain syndrome \\
& Neurotoxic medication \\
\hline Acquired & \\
& \\
& \\
& \\
& \\
& \\
& \\
&
\end{tabular}

The pathogenetic role of oxidative stress, inflammatory cytokines such as TNF- $\alpha$, and neuropeptides such as substance P (SP) are interesting to explore as a common final pathway in SFN in several immune-mediated inflammatory diseases. We described a patient with severe SFN who showed spectacular improvement after treatment with anti-TNF$\alpha$ therapy [20]. This case supports the idea that TNF$\alpha$ may be a crucial cytokine in the pathogenesis of SFN related to sarcoidosis and presumably in SFN related to other immune-mediated inflammatory diseases as well. Theoretical support for the effect of anti-TNF- $\alpha$ therapy on SFN may be found in the following. First, TNF- $\alpha$ plays an important role in immune-mediated neuropathies such as Guillain-Barré syndrome, in which small nerve fibers are also involved. Elevated serum concentration of TNF- $\alpha$ shows a positive correlation with neuropathy severity in patients with Guillain-Barré syndrome. Furthermore, the decrease in serum TNF- $\alpha$ and increase in serum soluble TNF receptors show a positive correlation with neuropathy recovery in those patients. Second, pharmacological and physiological studies report that proinflammatory cytokines such as TNF$\alpha$ are strongly involved in the generation and maintenance of neuropathic pain [21-25]. 
TABLE 3: Drugs for pain control in small fiber neuropathy.

\begin{tabular}{|c|c|c|}
\hline Drug & Dosage (per day) & Common side effects \\
\hline \multicolumn{2}{|l|}{ Antidepressants } & \multirow{5}{*}{$\begin{array}{l}\text { Sedation, weight gain, anticholinergic effects, sexual } \\
\text { dysfunction, arrhythmia (side effects most prominent } \\
\text { Sedation, weight gain, anticholinergic effects, sexual } \\
\text { dysfunction, arrhythmia (side effects most prominent } \\
\text { with amitriptyline) }\end{array}$} \\
\hline Amitriptyline (Elavil) & $20-150 \mathrm{mg}$ & \\
\hline Nortriptyline (Aventyl) & $20-150 \mathrm{mg}$ & \\
\hline Desipramine (Norpramin) & $20-200 \mathrm{mg}$ & \\
\hline Duloxetine (Cymbalta) & $60-120 \mathrm{mg}$ & \\
\hline \multicolumn{3}{|l|}{ Anticonvulsants } \\
\hline Gabapentin (Neurontin) & $600-3,600 \mathrm{mg}$ & Sedation, dizziness, peripheral edema, weight gain \\
\hline Pregabalin (Lyrica) & $150-600 \mathrm{mg}$ & Similar to gabapentin \\
\hline Topiramate (Topamax) & $25-400 \mathrm{mg}$ & $\begin{array}{l}\text { Weight loss, sedation, cognitive slowing, renal stones, } \\
\text { paresthesias }\end{array}$ \\
\hline Lamotrigine (Lamictal) & $25-400 \mathrm{mg}$ & $\begin{array}{l}\text { Stevens-Johnson syndrome, rash, dizziness, nausea, } \\
\text { sedation }\end{array}$ \\
\hline Carbamazepine (Tegretol) & $200-1,200 \mathrm{mg}$ & $\begin{array}{l}\text { Dizziness, sedation, ataxia, aplastic anemia, liver } \\
\text { enzyme elevation }\end{array}$ \\
\hline Oxcarbazepine (Trileptal) & $600-2,400 \mathrm{mg}$ & Dizziness, nausea, fatigue, leukopenia \\
\hline \multicolumn{3}{|l|}{ Topical anesthetics } \\
\hline 5\% Lidocaine patch (Lidoderm) & Every 12 hours & Local edema, burning, erythema \\
\hline 0.075\% Capsaicin patch & Three or four times a day & Burning \\
\hline \multicolumn{3}{|l|}{ Opioids, opioid agonists } \\
\hline Tramadol (Ultram) & $100-400 \mathrm{mg}$ & Sedation, dizziness, seizures, nausea, constipation \\
\hline Oxycodone (Oxycontin) & $10-100 \mathrm{mg}$ & $\begin{array}{l}\text { Sedation, constipation, nausea; potential for addiction } \\
\text { and abuse }\end{array}$ \\
\hline
\end{tabular}

\section{Treatment}

Although alternatives to corticosteroids have been frequently administered in this disease, corticosteroids remain the mainstay of treatment in sarcoidosis. Immunosuppressive agents (chlorambucil, cyclophosphamide, methotrexate, cyclosporine, azathioprine), anticytokine agents (thalidomide, pentoxifylline), antimalarials (chloroquine, hydroxychloroquine), melatonin, and monoclonal antibody (infliximab) have been used in chronic resistant sarcoidosis [26].

Usual treatments in sarcoidosis such as prednisone and methotrexate do not appear beneficial in sarcoidosis-related SFN (personal experience). SFN is disabling for patients and the pain is often difficult to treat. SFN has a high impact on the quality of life and often invalidates the patient. Case reports mention beneficial effects of intravenous immunoglobulin [19] and anti-TNF-alpha therapy [20]. The exact potency of these drugs needs further study, however.

Symptomatic neuropathic pain treatment in sarcoidosis patients is not different from treatment of neuropathic pain from other causes and consists of antidepressants, anticonvulsants and prolonged-release opioids (Table 3 ). However, in common with their effects in other neuropathic pain states, these agents provide limited pain relief in just 30-60\% of patients, at the cost of considerable side effects. These data indicate that there is an imminent need for analgesic agents with high efficacy in neuropathic pain patients without causing debilitating side effects.

\section{Directions for Future Studies}

As the role of TNF- $\alpha$ in the pathogenesis of SFN in sarcoidosis appears interesting to explore, anti-TNF therapy might be beneficial in the treatment of SFN in sarcoidosis. A recent therapeutic development has been the availability of agents that directly inactivate the proinflammatory cytokine TNF$\alpha$. Those are expensive drugs with possible severe side effects including opportunistic infection.

Recently, we initiated a program aimed at the treatment of neuropathic pain in patients with sarcoidosis with a novel therapeutic agent, ARA 290. ARA 290 is a nonhematopoietic erythropoietin analogue with potent anti-inflammatory and tissue protective properties, acting at the innate repair receptor [27-29]. In recent years, an endogenous system has been identified that antagonizes the production and action of proinflammatory cytokines that are involved in promoting tissue injury, while simultaneously activating repair processes. The primary mediator of this system is hypoglycosylated erythropoietin (EPO) that acts through a unique receptor isoform, the innate repair receptor (IRR), which is a combination of EPO and beta common receptor subunits. Many diverse preclinical models of tissue injury have demonstrated the efficacy of EPO as an effective cytoprotectant and activator of healing and repair. For example, EPO acting through the IRR has been shown to improve recovery and function from nerve injury in a variety of preclinical models, including the small-fiber neuropathy caused by uncontrolled 
diabetes [28]. Because the IRR has a lower affinity for EPO than the receptor utilized in hematopoiesis $(\sim 2 \cdot 0-20 \cdot 0 \mathrm{nM}$ versus $0 \cdot 2 \mathrm{nM}$ resp.), larger doses of erythropoietin must be administered to activate the IRR. Since EPO interacts with both of these receptors, translation of this knowledge into clinical use has been hindered by the presence of unavoidable hematopoietic side effects triggered by the hematopoietic receptor. For example, clinical studies evaluating use of EPO for tissue protection have consistently revealed increased rates of serious thrombosis [28]. To circumvent this problem, a number of IRR-specific ligands have been engineered.

One novel approach is pyroglutamate helix B surface peptide (ARA 290). This peptide mimics the spatial configuration of EPO that is believed to interact with the IRR. In spite of having a plasma half life of less than 2 minutes, ARA 290 is as efficacious as EPO in a wide variety of models of tissue injury. Additionally, preclinical toxicology studies of ARA 290 and single- and multiple-ascending repeated dosing of human volunteers and patients with kidney disease, diabetes mellitus, or sarcoidosis have raised no safety issues (unpublished data, Araim Pharmaceuticals).

First studies in animals (with nerve-damage induced neuropathic pain) and in patients with chronic neuropathic pain from sarcoidosis and diabetes mellitus indicated that ARA 290 is highly effective in causing pain relief in these neuropathic pain states. This compound appears potential for this chronic inflammatory disease and further investigation has been started.

\section{Abbreviations}

$\begin{array}{ll}\text { SFN: } & \text { Small-fiber neuropathy } \\ \text { TNF- } \alpha: & \text { Tumor necrosis factor- } \alpha \\ \text { NP: } & \text { Neuropathic pain } \\ \text { LD-SFSN: } & \text { length dependent small-fiber neuropathy } \\ \text { NLD-SFSN: } & \text { Non-length-dependent small-fiber } \\ & \text { neuropathy } \\ \text { IENFD: } & \text { Intra-epidermal nerve fiber density } \\ \text { QST: } & \text { Quantitive sensory testing } \\ \text { CDT: } & \text { Cold detection threshold } \\ \text { WDT: } & \text { Warm detection threshold } \\ \text { PHS: } & \text { Paradoxical heat sensation } \\ \text { MDT: } & \text { Mechanical detection threshold } \\ \text { VDT: } & \text { Vibration detection threshold } \\ \text { CPT: } & \text { Cold pain threshold } \\ \text { HPT: } & \text { Heat pain threshold } \\ \text { PPT: } & \text { Pain pressure threshold } \\ \text { MPT: } & \text { Mechanical pain threshold } \\ \text { LEP: } & \text { Laser evoked potential } \\ \text { CHEPs: } & \text { Contact heat evoked potentials. }\end{array}$

\section{References}

[1] U. Costabel and G. W. Hunninghake, "ATS/ERS/WASOG statement on sarcoidosis. Sarcoidosis statement committee. American thoracic society. European respiratory society. World association for sarcoidosis and other granulomatous disorders," European Respiratory Journal, vol. 14, pp. 735-737, 1999.
[2] S. Saidha, E. S. Sotirchos, and C. Eckstein, "Etiology of sarcoidosis: does infection play a role?" Yale Journal of Biology and Medicine, vol. 85, no. 1, pp. 133-141, 2012.

[3] N. J. Sweiss, K. Patterson, R. Sawaqed et al., "Rheumatologic manifestations of sarcoidosis," Seminars in Respiratory and Critical Care Medicine, vol. 31, no. 4, pp. 463-473, 2010.

[4] M. Bakkers, I. S. Merkies, G. Lauria et al., "Intraepidermal nerve fiber density and its application in sarcoidosis," Neurology, vol. 73, no. 14, pp. 1142-1148, 2009.

[5] E. Hoitsma, M. Marziniak, C. G. Faber et al., "Small fibre neuropathy in sarcoidosis," The Lancet, vol. 359, no. 9323, pp. 2085-2086, 2002.

[6] E. Hoitsma, J. P. Reulen, M. Baets De, M. Drent, F. Spaans, and C. G. Faber, "Small fiber neuropathy: a common and important clinical disorder," Journal of the Neurological Sciences, vol. 227, no. 1, pp. 119-130, 2004.

[7] E. Hoitsma, M. Drent, E. Verstraete et al., "Abnormal warm and cold sensation thresholds suggestive of small-fibre neuropathy in sarcoidosis," Clinical Neurophysiology, vol. 114, no. 12, pp. 2326-2333, 2003.

[8] J. Tavee and L. Zhou, "Small fiber neuropathy: a burning problem," Cleveland Clinic Journal of Medicine, vol. 76, pp. 297305, 2009.

[9] S. Khan and L. Zhou, "Characterization of non-lengthdependent small-fiber sensory neuropathy," Muscle and Nerve, vol. 45 , no. 1 , pp. 86-91, 2012.

[10] A. Hovaguimian and C. H. Gibbons, "Diagnosis and treatment of pain in small-fiber neuropathy," Current Pain and Headache Reports, vol. 15, no. 3, pp. 193-200, 2011.

[11] M. Bakkers, C. G. Faber, M. Drent et al., "Pain and autonomic dysfunction in patients with sarcoidosis and small fibre neuropathy," Journal of Neurology, vol. 257, no. 12, pp. 20862090, 2010.

[12] E. K. Krumova, C. Geber, A. Westermann, and C. Maier, "Neuropathic pain: is quantitative sensory testing helpful?" Current Diabetes Reports, vol. 12, no. 4, pp. 393-402, 2012.

[13] R. D. Treede, J. Lorenz, and U. Baumgartner, "Clinical usefulness of laser-evoked potentials," Neurophysiologie Clinique, vol. 33, no. 6, pp. 303-314, 2003.

[14] G. Lauria, D. R. Cornblath, O. Johansson et al., "EFNS guidelines on the use of skin biopsy in the diagnosis of peripheral neuropathy," European Journal of Neurology, vol. 12, no. 10, pp. 747-758, 2005.

[15] G. Devigili, V. Tugnoli, P. Penza et al., "The diagnostic criteria for small fibre neuropathy: from symptoms to neuropathology," Brain, vol. 131, no. 7, pp. 1912-1925, 2008.

[16] C. Han, J. G. Hoeijmakers, H. S. Ahn, P. Zhao, P. Shah, G. Lauria et al., "Nav1.7-related small fiber neuropathy: impaired slow-inactivation and DRG neuron hyperexcitability," Neurology, vol. 78, no. 21, pp. 1635-1643, 2012.

[17] R. P. Baughman, E. E. Lower, and M. Drent, "Inhibitors of tumor necrosis factor (TNF) in sarcoidosis: who, what, and how to use them," Sarcoidosis, Vasculitis and Diffuse Lung Diseases, vol. 25, no. 2, pp. 76-89, 2008.

[18] K. C. Gorson and A. H. Ropper, "Idiopathic distal small fiber neuropathy," Acta Neurologica Scandinavia, vol. 92, no. 5, pp. 376-382, 1995.

[19] J. G. Parambil, J. O. Tavee, L. Zhou, K. S. Pearson, and D. A. Culver, "Efficacy of intravenous immunoglobulin for small fiber neuropathy associated with sarcoidosis," Respiratory Medicine, vol. 105, no. 1, pp. 101-105, 2011.

[20] E. Hoitsma and C. G. Faber M, "Improvement of small fiber neuropathy in a sarcoidosis patient after treatment with infliximab," Sarcoidosis, Vasculitis, and Diffuse Lung Diseases, vol. 23, no. 1, pp. 73-77, 2006. 
[21] C. Sommer and M. Schafers, "painful mononeuropathy in C57BL/Wld mice with delayed wallarian degeneration: differential effects of cytokine production and nerve regeneration on thermal and mechanical hypersensitivity," Brain Research, vol. 784, no. 1-2, pp. 154-162, 1998.

[22] M. Schafers, C. Geis D Brors, T. L. Yaksh, and C. Sommer, "Anterograde transport of tumor necrosis factor-alpha in the intact and injured rat sciatic nerve," The Journal of Neuroscience, vol. 22, no. 2, pp. 536-545, 2002.

[23] A. Opree and M. Kress, "Involvement of the proinflammatory cytokines tumor necrosis factor-alpha, IL-1 beta, and IL- 6 but not IL- 8 in the development of heat hyperalgesia: effects on heat evoked calcitonin gene-related peptide release from rat skin," The Journal of Neuroscience, vol. 20, no. 16, pp. 62896293, 2000.

[24] M. Empl, S. Renaud, B. Erne et al., "TNF-alpha expression in painful and non-painful neuropathies," Neurology, vol. 56, no. 10, pp. 1371-1377, 2001.

[25] F. Q. Cunha, S. Poole, B. B. Lorenzetti, and S. H. Ferreira, "The pivotal role of tumour necrosis factor alpha in the development of inflammatory hyperalgesia," British Journal of Pharmacology, vol. 107, no. 3, pp. 660-664, 1992.

[26] P. Fazzi, "Pharmacotherapeutic management of pulmonary sarcoidosis," American Journal of Respiratory and Critical Care Medicine, vol. 2, no. 4, pp. 311-320, 2003.

[27] M. Brownlee, H. Vlassara, and A. Cerami, "Nonenzymatic glycosylation and the pathogenesis of diabetic complications," Annals of Internal Medicine, vol. 101, no. 4, pp. 527-537, 1984.

[28] M. Brines and A. Cerami, "Erythropoietin-mediated tissue protection: reducing collateral damage from the primary injury response," Journal of Internal Medicine, vol. 264, no. 5, pp. 405-432, 2008.

[29] M. Swartjes, A. Morariu, M. Niesters et al., "ARA290, a peptide derived from the tertiary structure of erythropoietin, produces long-term relief of neuropathic pain: an experimental study in rats and beta-common receptor knockout mice," Anesthesiology, vol. 115, pp. 1084-1092, 2011. 


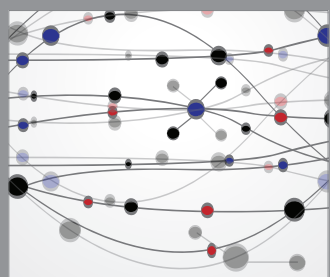

The Scientific World Journal
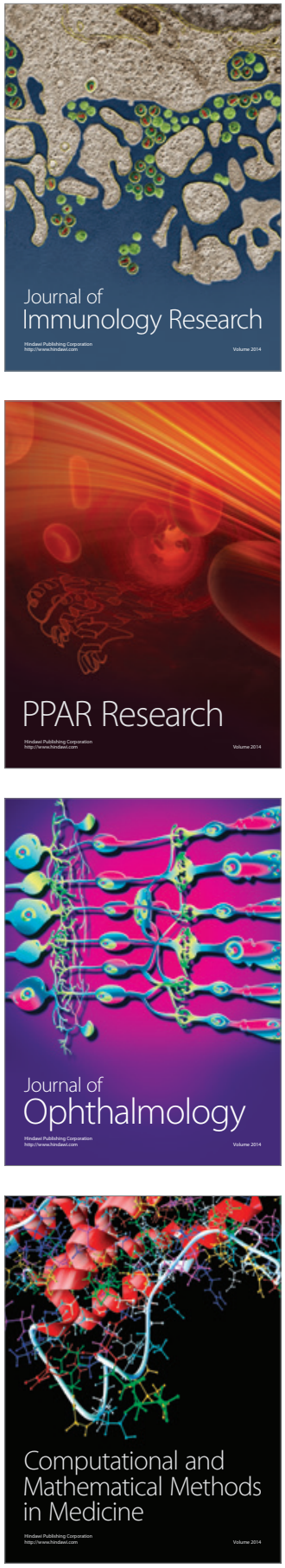

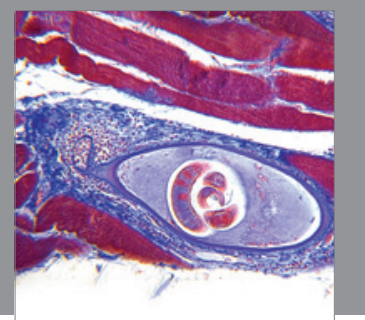

Gastroenterology

Research and Practice
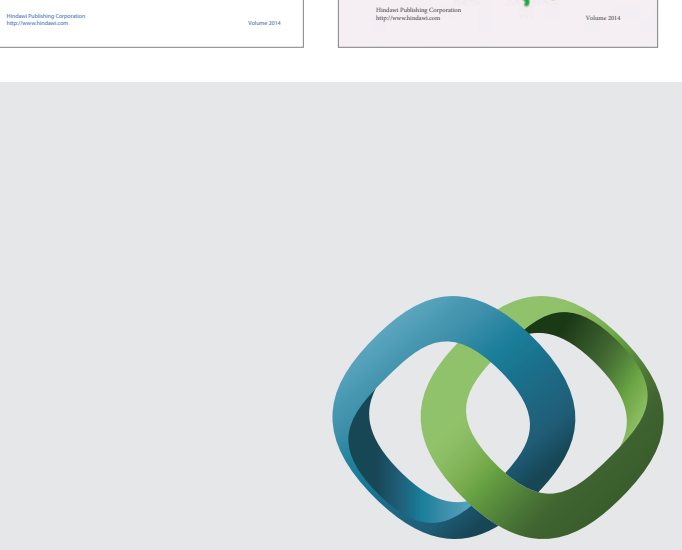

\section{Hindawi}

Submit your manuscripts at

http://www.hindawi.com
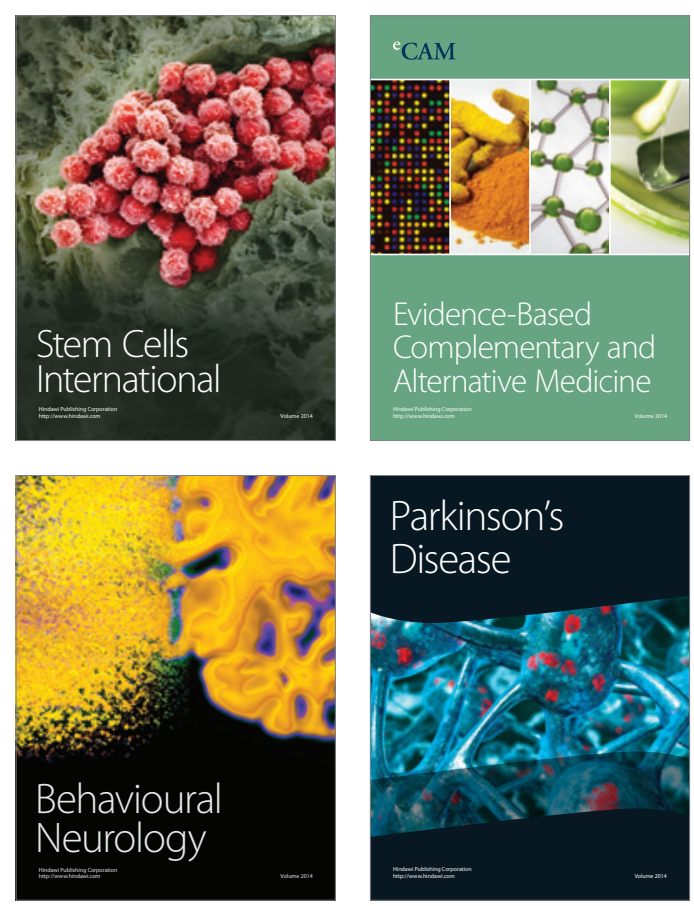

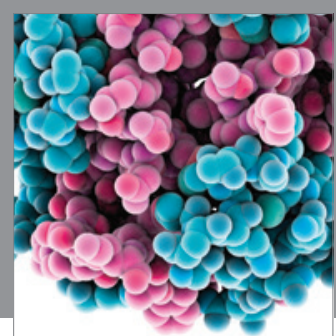

Journal of
Diabetes Research

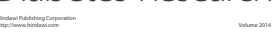

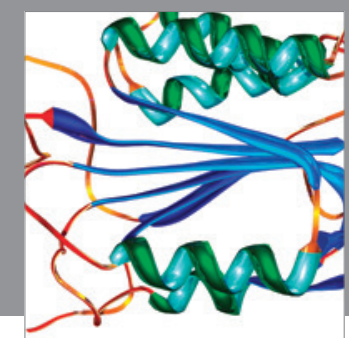

Disease Markers
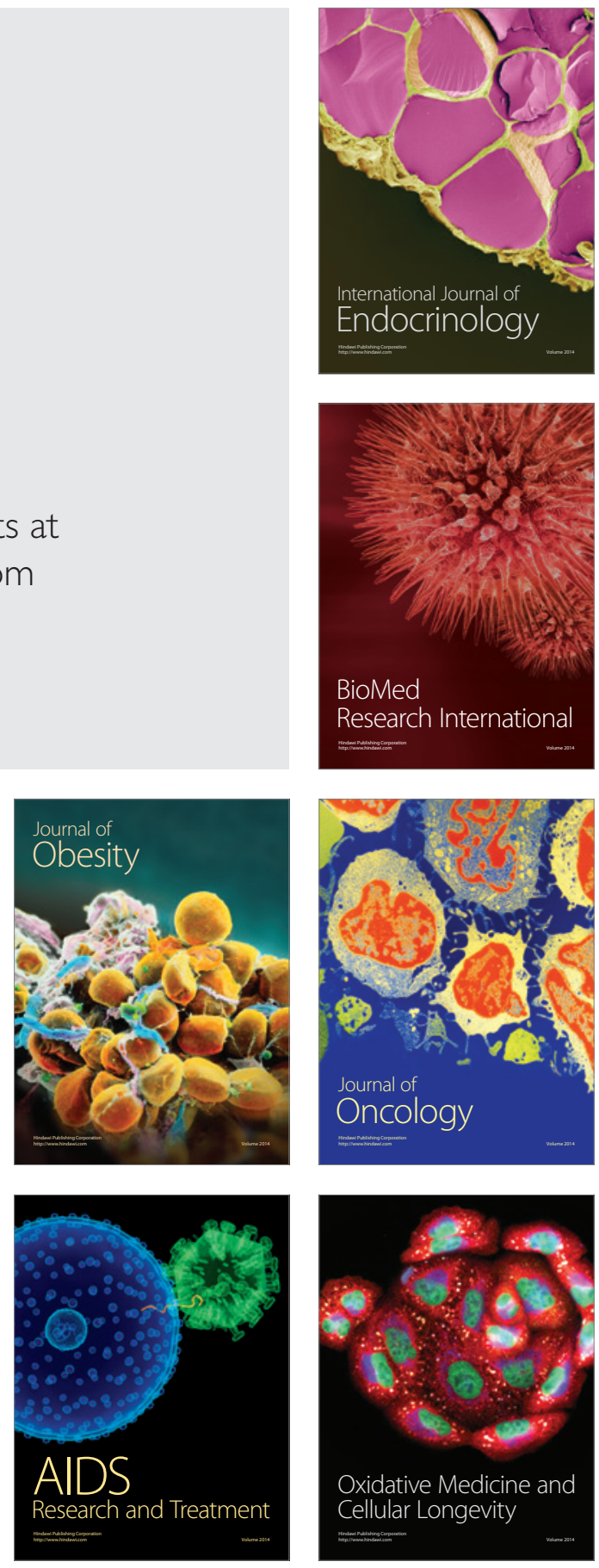